\title{
Estructura de razas, cruzas y criollos de ganado bovino en los sistemas productivos de la región Tulijá-Tseltal-Chol en Chiapas, México
}

\author{
Velázquez Avendaño, J. ${ }^{1}$ y Perezgrovas Garza, R. ${ }^{2}$
}

Unidad Académica Multidisciplinaria de Yajalón. Licenciatura en Desarrollo Sustentable. Universidad Intercultural de Chiapas. San Cristóbal de Las Casas. Chiapas, México.

${ }^{2}$ Instituto de Estudios Indígenas. Universidad Autónoma de Chiapas

\section{PaLABRAS CLAVE ADICIONALES}

Tipología de productores.

Superficie.

Campesinos.

Indígenas.

Análisis multivariado.

\section{RESUMEN}

Ante una escasa contribución de productos ganaderos de la región XIV Tulijá-Tseltal-Chol a la producción pecuaria de Chiapas, se realizó un estudio con el propósito de reconocer los tipos raciales de ganado bovino que conforman el arreglo estructural de los hatos ganaderos vinculado a una descripción de las características del sistema ganadero que apuntala la producción regional a fin de contar con información de utilidad que contribuya a comprender su funcionamiento, visualizando sus ventajas y desventajas y configurar que componentes son los que inciden para que exista esta baja producción a fin de desarrollar estrategias que fomenten su productividad. Se abordó el análisis calculando el porcentaje de inclusión de razas y/o de cruzamientos en los hatos, a lo cual se sumó el análisis de variables socioeconómicas: edad, nivel de estudio y variables productivas: población total de ganado, cantidad de vacas, nacimiento de becerros, tipo de pastos, extensión de potreros, número de potreros, mejoramiento de pastizales, desarrollo tecnológico, infraestructura disponible. Las variables fueron examinadas empleando el análisis de componentes principales, prueba de Bartlett y $\mathrm{KMO}$, las cuales indicaron valores satisfactorios para proceder con la prueba. Los resultados indican que el cruzamiento (entendido como el apareamiento entre bovinos de diferentes razas), es de las actividades reproductivas más utilizadas $(43,5 \%)$ siendo en las superficies menores donde mayor impacto tienen $(30,7 \%)$, aunque también es una acción muy socorrida en las superficies de mayor extensión, lo cual indica que el tamaño del predio no determina la selección de una raza pura para incrementar la producción. La presencia de ganado criollo se observa en superficies pequeñas $(3,2 \%)$, en el resto prácticamente ha desaparecido. Los grupos analizados y descritos comparten elementos comunes y son el sustento del sistema de razas y cruzamientos que impera en la región; no obstante, se debe resaltar la existencia de un contexto genético diverso asentado en todos los agrupamientos por lo que se puede inferir que la elección de una raza u otra, o la opción del cruzamiento no está influido por el tamaño del predio, ni por la edad o experiencia, desarrollo tecnológico o nivel de estudio, más bien parece que la relevancia de mantener el cruzamiento como factor productivo preferencial a una raza pura está condicionado por otro tipo de decisiones que apuntan hacia el mantener animales genéticamente resistentes o rústicos que permitan su sobrevivencia en condiciones geográficas y climáticas adversas y ligado posiblemente al modo de vida campesino indígena.

\section{Structure of breeds, crosses and Creole cattle under production systems in Tulijá- Tseltal-Chol region in Chiapas, Mexico}

\section{SUMMARY}

Due to a reduced contribution of the livestock producers from the XIVth Tulijá-TseltalChol Region to the overall livestock activity in Chiapas, a study was carried out in order to recognize the bovine cattle breed types that conform the structural arrangement of cattle herds linked to a description of the farming system characteristics that supports the regional production in order to be able to count on useful information which may help to understand its operation, visualising its advantages and disadvantages and to configure which components are the ones which may lower their production and to develop strategies that promote their productivity. The study was carried out by calculating the proportion of bovine pure breeds or its crosses within the different farm areas dedicated for livestock production, analyzing a series of socioeconomic variables such as: age and study level, other production variables were also studied: Total livestock population, number of cows, calves' birth, pasture type, pasture extent, number of pastures, rangeland improvement, technological development, available infrastructure. The variables were examined through statistical multivariate analyses by using the analysis of the main components and cluster analysis, Bartlett and KMO statistics, which indicated satisfactory values to proceed with the analyses. The results indicate that continuous crossbreeding is the most commonly used reproductive activity within the livestock production units in the region $(43.5 \%)$, being the smaller farms where it has a greater impact $(30.7 \%)$, although it is also a common practice in larger farms, indicating that the 
farm size does not determine the selection of a purebred in order to increase production. The presence of Creole cattle can be observed in small-size farms (3.2\%) while it has practically disappeared in the rest. From the analyzed and described groups we can conclude that they share common elements and can be used as a systematic tool to examine the livestock activity that prevails in the region; nevertheless, the existence of a diverse genetic background seated in all groupings should be noted, so we can infer that the selection of one race or another and the continuous crossbreeding is not influenced by the size of the farm, age, study level or experience of the producer, or the technological development; it would rather seem that the importance of maintaining crossbreeding as the preferred productive factor over pure breeds is conditioned by other decisions that point out towards keeping animals that show rusticitry and are genetically resistant, which enables survival under adverse geographical and climatic conditions also linked to the indigenous and peasant way of life.

\section{INTRODUCCIÓN}

La región XIV Tulijá-Tseltal-Chol en el estado de Chiapas, al sureste del territorio mexicano presenta un interesante contraste climatológico y geográfico desde el punto de vista de la producción ganadera con climas que van desde los muy fríos en la región de Tumbalá que alcanza escarpados a más de 1500 metros sobre el nivel del mar como los cálidos del Valle del Tulijá a 19 msnm por lo que los agrosistemas regionales ofrecen un entorno heterogéneo para el desarrollo de razas bovinas, con fuente de alimentación que van desde los alternativos susceptibles de ser aprovechados como el chanté (Gliricidia sepium) y las acacias (Acacia spp) que son fuentes de nutrientes de bajo costo (Perezgrovas, 1999), como los pastos nativos y los introducidos en sistemas extensivos de pastoreo. Sin embargo, se desconoce el estado actual que guarda el sistema productivo ganadero y la diversidad de razas que existen en la región y que pueden afectar la respuesta productiva del ganado al transferir diversas aptitudes, en algunos casos en detrimento de otras como, por ejemplo, la aptitud lechera (Orantes, 2010).

La ganadería bovina es uno de los recursos que forman la base de los sistemas productivos vigentes en la región XIV si consideramos que en el $88 \%$ de las unidades de producción agropecuaria permea y atraviesa está actividad combinada con actividades de tipo agrícola como el café, maíz, frijol y otros productos en un contexto biodiverso, ocupando un poco más de la mitad de la superficie de uso agropecuario (Velázquez, 2015), desde modelos de producción de tipo campesino indígena hasta el modelo pre-empresarial (García y Calles, 1988), que aquí subsisten. No obstante, su contribución a la producción estatal es de apenas 1,5\%, una de las más bajas del estado de Chiapas. En este contexto, la identificación de las razas de ganado que son manejados en estos agrosistemas, y la evaluación de los componentes que apuntalan este sistema, que sostienen estos niveles de producción, es una de las premisas de este estudio considerando que son la base del hato ganadero y por lo tanto se supone responsables en buena medida de su capacidad de producción ya que la interacción entre los tipos raciales y los demás componentes del sistema ganadero tiene su efecto en la productividad.
El presente trabajo deriva de estas consideraciones y propone hacer una contribución al conocimiento de los sistemas productivos ganaderos de esta región a través del estudio de la diversidad de biotipos de ganado bovino que conforman los hatos que perviven en la región XIV Tulijá-Tseltal-Chol aunado al análisis de indicadores de los componentes principales del sistema ganadero que permita evaluar las condiciones del sistema actual (Quiroga, 2001), en un primer acercamiento para entender la relación que guardan los productores ganaderos con sus animales y con el entorno productivo, reconociendo en el marco de esta primera aproximación los elementos que influyen en la toma de decisiones para escoger una raza $\mathrm{u}$ otra o bien optar por sus cruzamientos (entendido en esta región como el resultado del apareamiento entre razas diferentes) y que son la base de los hatos ganaderos de la región que hasta ahora ha sido muy poco analizado, en un proceso sistemático de reconocimiento de la realidad que en ellos ocurre.

\section{MATERIAL Y MÉTODOS}

Considerando que las razas de ganado bovino que se observan en las superficies dedicadas a la ganadería en la región XIV Tulijá-Tseltal-Chol suelen ser, además de multirraciales, el componente animal principal de los sistemas productivos vigentes en la región de estudio, complementados con otros componentes de tipo agrícola como el café y la milpa, lo cual le transfiere características muy particulares, por lo que se infiere que el sistema es multivariado (Valerio et al., 2004). Lo anterior sustenta la aplicación de un modelo de estudio basado en la metodología de análisis de componentes principales (ACP) que permita analizar las diversas variables exploradas y facilite el análisis de la variedad de razas o sus cruzamientos presentes y la relación que guardan tanto con variables productivas como sociales que derivan de un modo de vida (Hart, 1985; Rodríguez, 1993). Debe considerarse que el medio rural se conforma mayoritariamente de indígenas de origen mayense, localizados hacia el poniente los Tseltales, al suroriente Choles y al nororiente por Tsotsiles mientras que las poblaciones urbanas más importantes se conforman por mestizos y descendientes europeos que llegaron a estas regiones a principios del siglo XX (De Vos, 1998). Las actividades de este estudio iniciaron a partir de noviembre de 2012 hasta mayo de 2013 periodo durante el cual se hicieron recorridos de campo por las diversas unidades de producción pecuaria elegidas al azar, en los municipios de Salto de Agua, Tila, Tumbalá, Sabanilla, Yajalón, Chilón y Sitalá que conforman la región XIV los cuales están ubicados entre los $16^{\circ} 04^{\prime}$ y $17^{\circ} 56^{\prime}$ latitud $N$, y entre los $90^{\circ} 22^{\prime}$ y $92^{\circ} 42^{\prime}$ longitud $\mathrm{O}$, a una altitud que va desde los 19 a $1413 \mathrm{~m}$ sobre el nivel del mar (CEIEG, 2012). La región tiene una extensión de $4673,01 \mathrm{~km}^{2}$ y presenta varios tipos de clima: en las partes bajas de Salto de Agua es húmedo con lluvias todo el año, mientras que en las partes más altas de Tumbalá presenta clima templado subhúmedo con lluvias en verano (CEIEG, 2012). Las actividades económicas más preponderantes de la región son la agrícola, ganadera, forestal y turística. 
La investigación consideró un marco muestral de 6508 productores de bovinos registrados en el censo ganadero de la región (INEGI, 2013), utilizando un muestreo aleatorio simple, tomando en cuenta a cada unidad de producción pecuaria (UPP) como una unidad experimental, la cual está representada por cada productor ganadero. La muestra seleccionada resultó de aplicar la fórmula propuesta por Hernández et al. (2001) que determinó un tamaño de 324 productores seleccionados al azar y a quienes se les aplicó una encuesta socioeconómica y productiva.

$$
\mathrm{n}=\frac{N}{N d^{2}+1}
$$

Dónde:

$$
\begin{aligned}
& N=\text { tamaño de población, } \\
& d=\text { precisión }(5,5 \%), \\
& \mathrm{n}=\text { tamaño de la muestra. }
\end{aligned}
$$

Las variables estudiadas por medio de las encuestas fueron 53 las cuales fueron sistematizadas y analizadas por medio de ecuaciones estadísticas multivariadas, considerando como indicadores aquellos que permitieron un mejor análisis de los componentes animales, así como los sociales, que se estudiaron inicialmente por el método de reducción de dimensiones (Morrison, 1976) con el fin de resumir y explicar la información contenida en el conjunto de variables observadas. Las variables consideradas para el estudio fueron: tipo racial (se considera un componente importante de análisis que además de permitir cuantificar las razas de ganado presentes en la región, sirvió para explicar la relación que guarda con otros componentes del sistema ganadero); tamaño total de las superficies (reconoce y conjunta por tamaño la cantidad total de UPP's) superficie ganadera, población ganadera total, población de vacas, vacas en la ordeña, sementales, reposiciones, becerros, nacimiento de becerros, número de potreros, tamaño de los potreros y tipo de pastos. Otras variables analizadas de tipo productivo para una respuesta cualitativa fueron: UPP's que realizan ordeña, tecnología para la ordeña, corrales (disponibilidad de infraestructura), tipo de corral, tipo de pastos (y sus combinaciones en los potreros), disponibilidad de agua, disponibilidad de implementos agrícolas, tipos de implementos agrícolas, equipo para proporcionar sales, venta de becerros (motivos que obligan la venta) y norma oficial mexicana (participación en las campañas nacionales para erradicar la brucelosis y tuberculosis). De igual manera se estudiaron variables de tipo social como la edad y nivel educativo.

El procedimiento estadístico inició con la correlación de las variables, estadística de frecuencia y descriptiva a fin de estandarizarlas, posteriormente se aplicó la prueba KMO y de Bartlett, las cuales fueron útiles para definir el grado de estandarización (Ruiz et al. 2012). Las primeras pruebas coadyuvaron a confirmar que las variables seleccionadas son viables para desarrollar el estudio. Posteriormente se utilizó el análisis de componentes principales (ACP) y el de Conglomerados, que permitieron identificar las razas y la relación que guardan con el tamaño de las superficies, el nivel educativo, edad y otras variables productivas (Johnson, 2000).

Con el ACP se obtuvieron las comunalidades y la prueba de varianza total que contribuyó a registrar el número de componentes mínimos que permitieran elaborar la matriz de componentes y la prueba de rotación con el método de normalización varimax; posteriormente se hizo el análisis de conglomerados con la vinculación de Ward y al final realizar la tabla que resume los diversos conjuntos. El primero permitió reconocer que las variables elegidas explicaban satisfactoriamente los diversos conjuntos agrupados, y el segundo reveló las concentraciones en los datos para un agrupamiento eficiente que finalmente derivaron en la clasificación de diversos grupos en uso de las razas identificando sus diferencias. Finalmente el Dendrograma confirmó los agrupamientos que se conformaron a partir del estadístico utilizado. Los análisis se realizaron en el programa estadístico SPSS versión 19 (SPSS, 2010).

\section{RESULTADOS Y DISCUSIÓN}

El resultado final reconoce, por un lado, la diversidad de razas y cruzamientos que permea en los diferentes sistemas productivos y por otro, valora la situación que guardan los otros componentes del sistema para explicar cómo influyen en la productividad del sistema pecuario de esta región y si éstos, incluso los no productivos, determinan o influyen en los productores para decidir qué tipo de raza o cruzamientos mantener en sus hatos. Los resultados del análisis estadístico multivariado mostraron que las variables estudiadas mantienen una correlación independiente

Tabla I. Resultados de la prueba KMO y esfericidad de Bartlett aplicado al conjunto de datos de las unidades experimentales (Results KMO and Bartlett sphericity test applied to the data set of the experimental units).

\begin{tabular}{llc}
\hline Medida de adecuación & muestral de Kaiser-Meyer-Olkin. & 0,820 \\
\hline \multirow{2}{*}{$\begin{array}{l}\text { Prueba de esfericidad } \\
\text { de Bartlett }\end{array}$} & Chi-cuadrado aproximado & 2071,689 \\
& Sig. & 66 \\
\hline
\end{tabular}

Tabla II. Comunalidades formadas a partir de las variables estudiadas (Commonalities formed from the studied variables).

\begin{tabular}{lcc}
\hline & Inicial & Extracción \\
\hline Edad & 1.000 & 0,532 \\
Superficie total & 1.000 & 0,524 \\
Superficie ganadera & 1.000 & 0,829 \\
Población ganadera total & 1.000 & 0,871 \\
Vacas & 1.000 & 0,836 \\
Sementales & 1.000 & 0,560 \\
Reposiciones & 1.000 & 0,626 \\
Becerros & 1.000 & 0,517 \\
Potreros & 1.000 & 0,752 \\
Tamaño de los potreros & 1.000 & 0,557 \\
Nacimiento de becerros & 1.000 & 0,767 \\
Vacas en ordeña & 1.000 & 0,520 \\
\hline
\end{tabular}


como se puede ver en la adecuación muestral de KMO y la prueba de esfericidad de Bartlett y chi cuadrado aproximada, que ascendió a 0,820 lo cual indica que es aceptable para este tipo de estudios (tabla I). Por otro lado, el resultado de las comunalidades (tabla II) y de varianza total confirma que las variables son suficientes para explicar satisfactoriamente los factores comunes (tabla III), ya que por un lado la extracción mínima de datos no fue inferior a 0,5 y la varianza total observa que más del 81 \% es explicada con cinco componentes. El procedimiento de reducción de factores permitió visualizar el comportamiento de las variables y determinó los factores en los que se pueden agrupar.

La tabla IV muestra la estadística descriptiva de las variables en la que se observa para la variable superficie una media de 20 has y una mediana de 12 hectáreas. A partir de un estudio monográfico del censo agropecuario 2007 editado por la Universidad de Guadalajara y el INEGI (2012), señalan que el tamaño de las superficies en México pueden arreglarse, con fines de análisis, en tres: (1) reportan que casi el 90\% de las unidades de producción tienen superficies hasta de 20 hectáreas, (2) los grandes de más de 100 hectáreas corresponden al 2,2\% y el resto ubica a los medianos entre mayores de 20 hasta 100 has. En este estudio, se encontró que el 65,4\% de las UPP's son menores de 20 has, las superficies grandes el 1,5\% y el resto a las medianas (tabla V) observándose numéricamente una concentración menor de las superficies pequeñas que lo reportado a nivel nacional por la Universidad de Guadalajara. Este dato es interesante desde la perspectiva del modelo de producción campesino ganadero de la región de estudio, que en general, se encuentra en superficies menores de 20 hectáreas (Velázquez, 2015).

Por otro lado, a partir del análisis clúster los resultados del dendograma (figura 1), muestran la similitud en tres conglomerados al cortarse a una distancia de aglomeración no mayor de 10, los cuales explican de manera aceptable los datos conjuntados. En la tabla V se visualiza las características de cada conglomerado identificados como conglomerado 1,2 y $3(\mathrm{C} 1, \mathrm{C} 2, \mathrm{C} 3)$, en el que se destaca la presencia del ganado criollo asociado al primer agrupamiento que también se caracteriza por agrupar superficies pequeñas cuya media se ubicó en 11 hectáreas, lo cual señala que este tipo de ganado pervive principalmente en el sistema o modelo

Tabla III. Varianza total explicada de las variables analizadas por ACP (Total variance explained by the variables analyzed by ACP).

\begin{tabular}{|c|c|c|c|c|c|c|c|c|c|}
\hline \multirow{2}{*}{ Componente } & \multicolumn{3}{|c|}{ Autovalores iniciales } & \multicolumn{3}{|c|}{$\begin{array}{l}\text { Sumas de las saturaciones al cuadrado } \\
\text { de la extracción }\end{array}$} & \multicolumn{3}{|c|}{$\begin{array}{c}\text { Suma de las saturaciones al cuadrado } \\
\text { de la rotación }\end{array}$} \\
\hline & Total & $\begin{array}{l}\% \text { de la } \\
\text { varianza }\end{array}$ & $\begin{array}{c}\% \\
\text { acumulado }\end{array}$ & Total & $\begin{array}{c}\% \text { de la } \\
\text { varianza }\end{array}$ & $\begin{array}{c}\% \\
\text { acumulado }\end{array}$ & Total & $\begin{array}{c}\% \text { de la } \\
\text { varianza }\end{array}$ & $\begin{array}{c}\% \\
\text { acumulado }\end{array}$ \\
\hline 1 & 5.356 & 44,632 & 44,632 & 5.356 & 44,632 & 44,632 & 4.594 & 38,281 & 38,281 \\
\hline 2 & 1.494 & 12,447 & 57,079 & 1.494 & 12,447 & 57,079 & 2.025 & 16,876 & 55,157 \\
\hline 3 & 1.042 & 8,686 & 65,765 & 1.042 & 8,686 & 65,765 & 1.273 & 10,608 & 65,765 \\
\hline 4 & 0,996 & 8,296 & 74,061 & & & & & & \\
\hline 5 & 0,910 & 7,583 & 81,644 & & & & & & \\
\hline 6 & 0,644 & 5,367 & 87,010 & & & & & & \\
\hline 7 & 0,509 & 4,241 & 91,252 & & & & & & \\
\hline 8 & 0,425 & 3,541 & 94,792 & & & & & & \\
\hline 9 & 0,214 & 1,781 & 96,573 & & & & & & \\
\hline 10 & 0,151 & 1,258 & 97,831 & & & & & & \\
\hline 11 & 0,145 & 1,206 & 99,037 & & & & & & \\
\hline 12 & 0,116 & 0,963 & 100,000 & & & & & & \\
\hline
\end{tabular}

Tabla IV. Resultado del análisis estadístico descriptivo de las variables estudiadas (Outcome of descriptive statistical analysis of the studied variables).

\begin{tabular}{|c|c|c|c|c|c|c|}
\hline & $\mathrm{N}$ & Rango & Mínimo & Máximo & Media & Desv. típ. \\
\hline Edad & 323 & 69 & 19 & 88 & 48,30 & 14,574 \\
\hline Superficie total & 324 & 278 & 2 & 280 & 20,90 & 26,858 \\
\hline Superficie ganadera & 324 & 120 & 1 & 120 & 13,09 & 14,712 \\
\hline Población ganadera total & 324 & 283 & 2 & 285 & 27,83 & 31,604 \\
\hline Vacas & 323 & 150 & 0 & 150 & 13,89 & 16,074 \\
\hline Semental & 323 & 60 & 0 & 60 & 1,29 & 3,424 \\
\hline Reposiciones & 319 & 80 & 0 & 80 & 5,10 & 7,232 \\
\hline Becerros & 323 & 50 & 0 & 50 & 6,93 & 8,120 \\
\hline Potreros & 323 & 37 & 1 & 38 & 3,45 & 3,350 \\
\hline Tamaño de los potreros & 294 & 30 & 1 & 30 & 3,82 & 3,865 \\
\hline Nacimiento de becerros & 323 & 100 & 0 & 100 & 7,98 & 9,577 \\
\hline Vacas en ordeña & 322 & 25 & 0 & 25 & 0,44 & 2,741 \\
\hline N válido (según lista) & 283 & & & & & \\
\hline
\end{tabular}


Tabla V. Conformación de los conglomerados y comportamiento de las variables estudiadas en la región XIV Tulijá-Tseltal-Chol en Chiapas, México (Formation of clusters and abstract variables studied in the region XIV Tulijá-Tseltal-Chol in Chiapas, Mexico).

\begin{tabular}{|c|c|c|c|c|c|}
\hline \multirow{2}{*}{ Conceptos } & & & \multicolumn{3}{|c|}{ Conglomerados } \\
\hline & & & 1 & 2 & 3 \\
\hline \multicolumn{3}{|l|}{ Recuento (UPP ${ }^{1}$ ) } & 189 & 87 & 7 \\
\hline \multicolumn{3}{|l|}{$\%$ del $\mathrm{N}$ de la tabla } & $66,8 \%$ & $30,7 \%$ & $2,5 \%$ \\
\hline \multirow{2}{*}{ Superficie total (has) } & & 11 & 32 & 144 \\
\hline & \multicolumn{2}{|l|}{ Mediana } & 10 & 27 & 133 \\
\hline \multirow[t]{2}{*}{ Superficie ganadera (has) } & \multicolumn{2}{|l|}{ Media } & 7 & 22 & 61 \\
\hline & \multicolumn{2}{|l|}{ Media } & 14 & 49 & 151 \\
\hline \multirow[t]{3}{*}{ Población ganadera } & \multicolumn{2}{|l|}{ Máximo } & 36 & 35 & 140 \\
\hline & \multicolumn{2}{|l|}{ Mínimo } & 17 & 2 & 15 \\
\hline & \multicolumn{2}{|l|}{ Media } & 7 & 25 & 69 \\
\hline \multirow[t]{2}{*}{ Vacas (cabezas) } & \multicolumn{2}{|l|}{ Máximo } & 20 & 20 & 80 \\
\hline & \multicolumn{2}{|l|}{ Mínimo } & 8 & 0 & 0 \\
\hline Sementales & \multicolumn{2}{|l|}{ Media } & 0,9 & 2,2 & 2,4 \\
\hline Reposiciones & \multicolumn{2}{|l|}{ Media } & 2,6 & 8,5 & 25,0 \\
\hline Becerros & \multicolumn{2}{|l|}{ Media } & 3,4 & 13,5 & 17,6 \\
\hline Potreros (cantidad/UPP) & \multicolumn{2}{|l|}{ Media } & 2,7 & 4,4 & 14,4 \\
\hline Tamaño de potreros (has) ${ }^{2}$ & \multicolumn{2}{|l|}{ Media } & 3,1 & 5,4 & 5,0 \\
\hline Nacimientos (becerros/año) & \multicolumn{2}{|l|}{ Media } & 4,8 & 13,5 & 34,4 \\
\hline Vacas en ordeña (cabezas) & \multicolumn{2}{|l|}{ Media } & 0,1 & 1,1 & 1,4 \\
\hline \multirow[t]{8}{*}{ Edad del productor (años) } & \multicolumn{2}{|l|}{ Media } & 48 & 49 & 49 \\
\hline & \multirow{2}{*}{ Cebú } & Recuento & 21 & 7 & 0 \\
\hline & & $\%$ N/tabla & $7,4 \%$ & $2,5 \%$ & $0,0 \%$ \\
\hline & \multirow{2}{*}{ Charolai } & Recuento & 2 & 2 & 1 \\
\hline & & $\%$ N/tabla & $0,7 \%$ & $0,7 \%$ & $0,4 \%$ \\
\hline & \multirow{2}{*}{ Criollo } & Recuento & 7 & 2 & 0 \\
\hline & & $\%$ N/tabla & $2,5 \%$ & $0,7 \%$ & $0,0 \%$ \\
\hline & & Recuento & 87 & 34 & 2 \\
\hline Tipo racial & Cruzado & $\%$ N/tabla & $30,7 \%$ & $12,0 \%$ & $0,7 \%$ \\
\hline & & Recuento & 0 & 3 & 1 \\
\hline & Simbrah & $\%$ N/tabla & $0,0 \%$ & $1,1 \%$ & $0,4 \%$ \\
\hline & & Recuento & 10 & 6 & 2 \\
\hline & Simmental & $\%$ N/tabla & $3,5 \%$ & $2,1 \%$ & $0,8 \%$ \\
\hline & & Recuento & 62 & 33 & 1 \\
\hline & Suizo & $\%$ N/tabla & $21,9 \%$ & $11,7 \%$ & $0,4 \%$ \\
\hline
\end{tabular}

${ }^{1} \mathrm{UPP}=$ Unidad de producción pecuaria, equivalente a una unidad experimental. ${ }^{2}$ Promedio de hectáreas con pastos mejorados y nativos.

de producción campesino indígena, que es el sistema productivo más extendido en la región y que posee en promedio 9 hectáreas (Velázquez, 2015). Del porcentaje total de ganado existente en la región se encontró que el $43,5 \%$ de la superficies ganaderas tienen hatos con una composición basada en el cruzamiento de razas, aunque no se tienen datos precisos sobre las razas participantes, se supone que son multirraciales. El 33,9\% de las superficies tienen al ganado suizo como la raza base de sus hatos ganaderos, el ganado cebú el 9,9\%, Simmental el 6,0\% y el resto tienen Simbrah $(1,4 \%)$ y charoláis (1,8\%). Es interesante observar que el 3,2\% de los hatos mantienen ganado reconocido regionalmente como Criollo, es decir de los descendientes del ganado que llegó a México en la época de la colonia, sin que hasta ahora se reconozca puntualmente a qué tipo de ganado criollo se hace referencia. Esta información es valiosa en términos de productividad e indica que la tendencia hacia el cruzamiento también llamado regionalmente como mestizaje, es una práctica común en todos los tipos de sistemas de la región estudiada.
Estos resultados son semejantes a los obtenidos por Orantes (2010) en su estudio sobre razas bovinas en la Región I Centro del estado de Chiapas y con los mostrados por el ministerio de Agricultura y Ganadería de México (SAGARPA, 2010) que han reportado resultados semejantes.

Por otro lado, la suma de la población de vacas, sementales, reposiciones y becerros (en unidades animales, ua), encontró las medias siguientes: $\mathrm{C} 1=12$, $\mathrm{C} 2=40, \mathrm{C} 3=102$ ua mientras que la capacidad de carga por hectárea en esta región, de acuerdo a la Sagarpa, es menor a una unidad por hectárea que resulta en 1,7 ua/ha la carga animal que las superficies ganaderas mantienen en este momento lo cual es un indicio que existe un problema muy serio de sobrepoblación en los tres agrupamientos que tiene un efecto negativo para la producción, y se ahonda debido a que solo el 4,4\% de las superficies tienen alternativas como el pasto de corte y el 5,4\% utilizan equipo agrícola como picadora de pastura. Cabe aclarar, en relación a la carga animal 


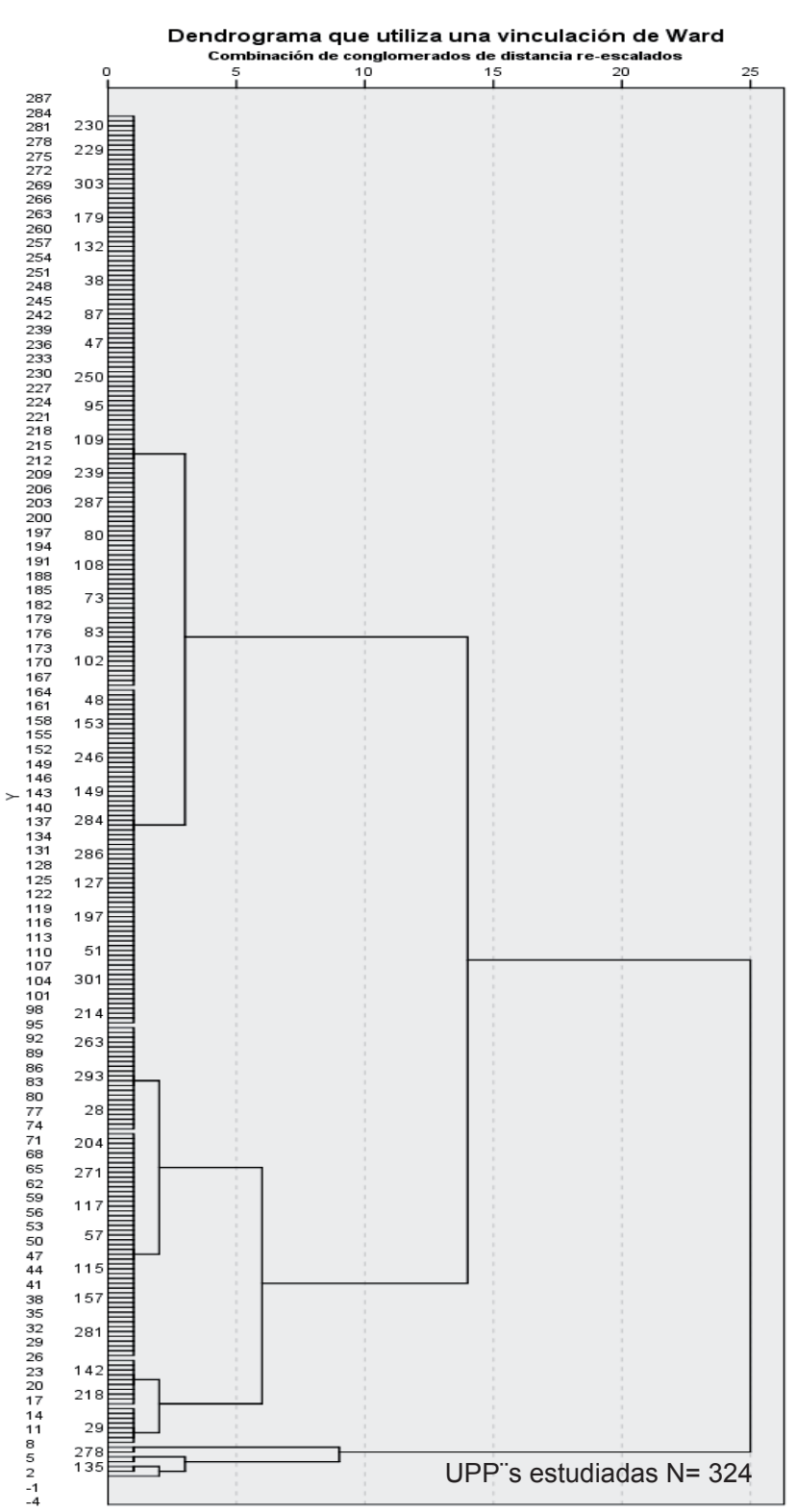

Figura 1. Combinación de conglomerados de distancia obtenidos de las unidades experimentales (Combination of distance clusters obtained from the experimental units). actual, que la cobertura con pastizales se encuentra de la siguiente manera: el 49,8\% de la superficie ganadera está cubierta totalmente con zacate estrella africana (Cynodon plectostachyus), y el 33,2\% es una combinación de estrella africana con otros pastos como el chontalpo o señal (Bracharia Decumbens cv. Basilisk), y el insurgente (Bracharia brizantha), el resto está cubierto en diversas combinaciones de otros pastos como el alemán (Echinochloa polystachya), gigante (Pennisetum purpureum), King grass (Saccarum sinense Roxb), Tanzania (Panicum maximum cv. Tanzania), zacatón (hyparrhenia rufa), y la grama nativa que además cubre totalmente los potreros del 5,3\% de las UPP's (tabla VI). Es importante resaltar que el $22 \%$ de las UPP's manifestaron tener problemas de escases de pastura en algún momento por lo que se ven obligados a la venta de sus becerros. El sistema de pastoreo que se perfila en la ganadería de la región comprende el pastoreo extensivo practicado en potreros cuyo rango en extensiones van desde una hectárea hasta los de 20 (media $C 1=3, C 2=5, C 3=5$ ) en la que el abastecimiento de agua se hace primordialmente de ríos y pozos y si bien la mayor parte de los hatos se les proporciona sal blanca o mineral estas se les proporciona incluso en canoas rústicas de madera o llantas improvisadas como saladeros.

Otro componente importante del sistema es la escasa producción de leche para abastecer la población humana en la que participan solo el 3,2\% de los hatos (en particular en los de superficies medianas con el $2,5 \%$ y el resto en ganaderías con superficies pequeñas), mediante ordeña tradicional manual, en corrales hechos de alambrado de púas y piso de tierra $(71,4 \%)$, y un poco más del $7 \%$ no cuenta con ellos. El baño garrapaticida se realiza a base de bombas de aspersión incluso en las ganaderías más grandes ya que menos del $1 \%$ tienen baños de inmersión. Por lo que se deduce que el sistema productivo está diseñado para la producción de cría, con venta de becerros recién destetados y que permea a través de todos los sistemas productivo vigentes en la región, con un desarrollo de tecnología y equipo en la mayoría de conjuntos agrupados sumamente escaso y pueden ser elementos que limitan a mantener un bajo nivel de producción. El promedio de edad de los tres conglomerados y el nivel

Tabla VI. Cobertura de pastizales encontrados en las superficies ganaderas de la región XIV Tulijá-TseltalChol en Chiapas, México (Coverage found on cattle grazing areas in the region XIV Tulijá-Tseltal-Chol in Chiapas, Mexico).

\begin{tabular}{|c|c|c|c|c|c|}
\hline \multirow{2}{*}{ Conceptos } & & & \multicolumn{3}{|c|}{ Conglomerados } \\
\hline & & & 1 & 2 & 3 \\
\hline \multirow{3}{*}{\multicolumn{2}{|c|}{$\begin{array}{l}\text { Recuento (UPP) } \\
\% \text { del N de la tabla }\end{array}$}} & & 189 & 87 & 7 \\
\hline & & & $66,8 \%$ & $30,7 \%$ & $2,5 \%$ \\
\hline & \multirow{2}{*}{ Estrella africana } & Recuento & 117 & 23 & 1 \\
\hline \multirow{11}{*}{ Tipo de pasto } & & $\%$ N/tabla & $41,3 \%$ & $8,1 \%$ & $0,4 \%$ \\
\hline & \multirow{2}{*}{ Estrella-brizantha-chontalpo } & Recuento & 31 & 24 & 3 \\
\hline & & $\%$ N/tabla & $11,0 \%$ & $8,5 \%$ & $1,1 \%$ \\
\hline & \multirow{2}{*}{ Estrella-grama natural } & Recuento & 8 & 6 & 0 \\
\hline & & $\%$ N/tabla & $2,8 \%$ & $2,1 \%$ & $0,0 \%$ \\
\hline & \multirow{2}{*}{ Grama natural } & Recuento & 14 & 2 & 0 \\
\hline & & $\%$ N/tabla & $4,6 \%$ & $0,7 \%$ & $0,0 \%$ \\
\hline & \multirow{2}{*}{ Brizantha-chontalpo } & Recuento & 11 & 17 & 1 \\
\hline & & $\%$ N/tabla & $3,9 \%$ & $6,0 \%$ & $0,4 \%$ \\
\hline & \multirow{2}{*}{ Otros } & Recuento & 11 & 15 & 2 \\
\hline & & $\%$ N/tabla & $3,9 \%$ & $5,3 \%$ & $0,7 \%$ \\
\hline
\end{tabular}


de estudio no resultó diferente entre los conjuntos, por lo que se puede inferir que tienen nula contribución para influir en la decisión de mantener un determinado tipo de raza en sus hatos ganaderos o inducir cambios al sistema productivo.

Con respecto a la presencia múltiple de razas llama la atención que la premisa fundamental para la introducción de razas en los sistemas ganaderos de la región ha sido el de incrementar los niveles de productividad; sin embargo, como ha quedado demostrado, el nivel productivo es de los más bajos en el estado y todo ello permea en todos los conglomerados en el que concurren ganados de todas las razas, desde el Suizo, Simmental hasta el charoláis y Simbrah y por supuesto el ganado que se conforma a partir de las cruzas de razas. En otras palabras, si bien las razas y cruzamientos aportan lo suficiente por su efecto aditivo no resuelven la problemática productiva ganadera regional ya que esta problemática responde a un conjunto de deficiencias analizadas más arriba, inmerso en un contexto, cabe recalcar por su relevancia, cultural y de cosmovisión del ganadero indígena, ya que es probable que también pueda estar relacionada con la cultura de la diversificación, no sólo de cultivos y de vida silvestre, características muy desarrolladas en los sistemas campesinos de esta región (Velazquez, 2015) como componentes que imprimen la dinámica de todo el sistema y que impactan en las expresiones culturales de estos pueblos, como también lo menciona Boege (2006), sino también de razas y cruzas de ganado bovino (SAGARPA, 2010), a quienes en un momento dado podría, incluso, parecerles poco relevante la pureza de una raza y en la búsqueda del acondicionamiento de sus animales a las zonas geográficas y climatológicas prefieran desarrollar el cruzamiento con el propósito de mejorar la adaptación.

Las características descritas para los grupos conformados son similares a los datos encontrados por Orantes (2014), en el sentido que las unidades de producción campesina (UPC) conforman un grupo importante de productores ganaderos con características específicas en el que predominan las cruzas de ganado. Por otro lado, Magdaleno et al. (2014) reportaron una experiencia similar de un conjunto de mucha importancia de ganaderos que producen en un contexto de producción campesina en un medio indígena en Acambay, Estado de México, en el centro del país.

En suma, el análisis de los agrupamientos del ganado bovino de la región XIV Tulijá-Tseltal-Chol en relación a los componentes del sistema ganadero, el tamaño de las superficies y la presencia de las razas indica que hay dos razas que podrían atribuirse ser consideradas las dominantes en un momento dado: suizo y cebú aunque se debe reconocer que existe una práctica de mayor magnitud que es el sistema de cruzamientos que permite deducir que en ellos descansa la productividad de los sistemas o que apuntala la producción de ganado bovino en la región compartiendo afinidades como la presencia de un patrón común característico de cruzamiento de las diferentes razas, en el cual se ha perdido en buena medida la presencia del ganado criollo como tal, aunque debe reconocerse que existe un número importante de superficies de los tres tamaños con productores con mayor especialización que pueden ser catalogados como los promotores o suscriptores de una raza pura.

\section{CONCLUSIONES}

Los grupos analizados y descritos contienen características y elementos comunes compartidos que son la base del sistema ganadero y de razas y cruzamientos que impera en la región, componentes importantes de la actividad pecuaria creadora de la riqueza económica, cultural y social que fluye de los sistemas productivos regionales. La ganadería regional comparte afinidades productivas muy interesantes y que permea en todos los conjuntos observados como es la práctica del sistema de crías, y no de doble propósito como podría esperarse en un momento dado, la diversidad de pasturas aunque asentado fuertemente en zacate estrella africana, en modelos extensivos de pastoreo con una sobrecarga de ganado que impacta negativamente el desempeño de los hatos agravado por el escaso desarrollo tecnológico en infraestructura y equipo para subsanar deficiencias como la escases de pasturas y en consecuencia contribuyen de manera conjunta a mantener la baja productividad regional. Planteamientos que convergen en un contexto de diversidad de razas y cruzas asentado en todos los agrupamientos, por lo que se puede inferir que la elección de una raza u otra, o bien optar por cruzamiento no dependen del tamaño de la superficie, edad o experiencia del productor, así como tampoco por el desarrollo tecnológico o nivel de estudio, ni tampoco son la alternativa para incrementar la producción por si solos y más bien la relevancia de mantener el cruzamiento como opción productiva a una raza pura está condicionado por otro tipo de decisiones que apuntan hacia la conservación de animales genéticamente resistentes o rústicos que permitan su sobrevivencia en condiciones geográficas y climáticas adversas, todo ello ligado al modo de vida campesino indígena de esta región.

\section{BIBLIOGRÁFIA}

Boege, E. 2006. Territorio y diversidad biológica, la agrobiodiversidad de los pueblos Indígenas de México. En biodiversidad y conocimiento tradicional en la sociedad rural. México. Centro de Estudios para el Desarrollo Rural Sustentable y Soberanía Alimentaria. Cámara de Diputados LIX Legislatura. Cámara de La Unión. 237 pp.

CEIEG (Comité Estatal de Información Estatal de Información Estadística y Geografía). 2012 y 2013. Gobiernos municipales/regiones. Estado de Chiapas. México.

De Vos, J. 1988. Oro verde, la conquista de la selva Lacandona por madereros tabasqueños, 1822-1949. FCE-Instituto de la Cultura de Tabasco. México. pp. 250-300.

García, H.C. y L.M. Calles. 1988. Consideraciones metodológicas para la tipificación de sistemas de producción bovina a partir de fuentes secundarias. Revista Corpoica 2: 6-16.

Hart, R.D. 1985. Agroecosistemas: Conceptos básicos. Centro Agronómico Tropical de Investigación y Enseñanza. Turrialba. Costa Rica. 159 pp. Hernández, R.; Fernández, C. y Baptista, P. 2001. Metodología de la investigación. $4^{a}$. Ed. México. Mc Graw Hill. New york. EU.

INEGI (Instituto Nacional de Estadística Geográfica e Informática). 2013. Censo Agrícola, Ganadero y Forestal. Estado de Chiapas. México.

Johnson, D. 2000. Métodos multivariados aplicados al análisis de datos. International Thomson Editores. México. 566 pp. 
Magdaleno, E.; Jiménez, M.; Martínez, T. y Cruz, B. 2014. Estrategias de las familias campesinas en Pueblo Nuevo, Municipio de Acambay, Estado de México. Agricultura, Sociedad y Desarrollo, 6: 167-179.

Morrison, D.F. 1976. Multivariate statistical methods. McGraw Hill Book Company. New york. Estados Unidos. 338 pp.

Perezgrovas, G.R. 1999. Los carneros de San Juan. Ovinocultura indígena en Los Altos de Chiapas. $2^{a}$ ed. Instituto de Estudios Indígenas-UNACH. San Cristóbal de Las Casas. Chiapas. México.

Orantes, M.A.; Platas, R.D.; Córdova, A.V.; De Los Santos, L. y Córdova, A.A. 2014. Caracterización de la ganadería de doble propósito en una región de Chiapas, México. Ecosistemas y recursos agropecuarios, Año 2, No. 4. Universidad Juárez Autónoma de Tabasco.

Quiroga, R. 2001. Indicadores de sostenibilidad ambiental y desarrollo sostenible: estado del arte y perspectivas. http://www.cepal.org/ es/publicaciones/5570-indicadores-de-sostenibilidad-ambientaly-de-desarrollo-sostenible-estado-del (05/11/2008).

Rodríguez, P. 1993. Sistemas de producción, conceptos y métodos de aplicación. En: Curso de especialización en interpretación de imágenes de sensores remotos aplicada a levantamientos rurales. IGAC. Bogotá, Colombia. pp. 40-69.

Ruiz, M.; J. Ruiz y C.J. Verena. 2012. Estudio de sistemas de producción de carne bovina en un municipio del estado de Hidalgo, México. Rev Cubana Cienc Agríc, 46: 261-265.

SAGARPA (Secretaría de Agricultura, Ganadería, Desarrollo Rural, Pesca y Alimentación). 2010. INIFAP (Instituto Nacional de Investigaciones Forestales, Agrícolas y Pecuarias). 2010. Informe Final. http//www. utep.inifap.gob.mx (18/09/2015).

SPSS. IBM SPSS 2010. User's guide: Statistics (version 19). SPSS Inc. NY. USA.

Valerio, D.C.; Acero, R.; García, J.M.; Castaldo, A.M. y Peinado, A. 2004. Metodología para la caracterización y tipificación de sistemas ganaderos. Producción animal y gestión. Vol. 1. Universidad de Córdoba. Argentina.

Velázquez, A.J. 2015. Tipología de productores de la región indígena XIV Tulijá-Tseltal-Chol, Chiapas. México. Rev Mex Cienc Pec, 6: 405-417. 